\title{
Bosques maduros y coleópteros saproxílicos, una visión desde Francia
}

\section{H. Brustel', J.M. Savoie ${ }^{1}$ y N. Estela i Ribera ${ }^{2}$}

I Ecole d’Ingénieurs de Purpan, 75 voie du TOEC, 3 I075 TOULOUSE cedex 3 Herve.brustel@ PURPAN.FR

2 Centro Iberoamericano de la Biodiversidad (CIBIO), Universidad de Alicante, Spain

\section{ABSTRACT}

Old growth forests are essential for biodiversity conservation, but they're becoming very rare, scattered and threatened throughout Europe. Old growth forest is a concept with many dimensions such as naturalness, stand maturity, native tree species and strictly associated biodiversity. Some stenotopic, flight less and rare saproxylic beetles allow us to evaluate historical forest continuity. However the saproxylic beetles species included in the Natura 2000 appendix are not the most relevant indicators for hot spot detection. We provide examples of saproxylic hot spots from France, Central Europe, Scandinavia and Macaronesia.

Keywords: Beetles, Natura 2000, Old growth forest, Wilderness, Conservation.

\section{LOS BOSQUES MADUROS}

En Francia, los bosques cubren en la actualidad 16 millones de hectáreas, lo que la sitúa en el tercer país de Europa, sin tener en cuenta a Rusia, después de Suecia y Finlandia. El bosque en Francia es, esencialmente, un bosque joven cuya superficie crece notablemente desde la segunda mitad del siglo XIX. Se estima que en 1830, la superficie ocupada por bosques era solamente entre 8,9 y 9,5 millones de hectáreas (CINOTTI, 1996). Sin tener en cuenta los cambios de uso acontecidos, más del $40 \%$ de la superficie boscosa francesas tiene menos de dos siglos, y es seguida por la dedicada al uso agrícola, que evidentemente conlleva muchas mas alteraciones sobre el suelo, las series florísticas y faunísticas (DUPOUEY et al., 2002). Sin embargo, los bosques viejos donde la influencia humana es escasa (bosques maduros) no representan, más de 30.000 ha (siendo el $0,2 \%$ de la superficie forestal total, BARTHOD, 1997), estando además muy fragmentada. Entre estos bosques franceses poco perturbados, solamente 15000 ha tienen hoy un estado de reserva integral. Estas cifras sitúan en el plano forestal a Francia muy por detrás de otros países de Europa occidental y central.

Estos bosques se clasifican, bajo conceptos que reflejan interpretaciones contrastadas: bosques viejos seminaturales (KIRBY, 1988), bosques salvajes (BRATTION \& ANDREWS, 1991), bosques muy viejos (BARTHOD, 1993), bosques subnaturales 
(BARTHOD \& TOUZET, 1994); PONTHUS, 1996), bosques casi naturales (BLANDIN, 1995), bosques primitivos (BARBALAT, 1995), bosques naturales (SCHNITZLER-LENOBLE, 2002), "bosques viejos" o "bosques ancianos" (traducción del concepto de "old-growth forests" de los autores anglosajones; SNÄLL, 1997; BURGMAN \& LINDENMAYER, 1998; SIPPOLA et al., 1998; MOSSELER et al., 2003; PERERA et al., 2003). La mayor parte de estos autores se han puesto de acuerdo para aplicar estas expresiones a las poblaciones forestales perturbadas por las actividades humanas a lo largo de su historia, pero que ya no son objeto de intervención desde hace algunas décadas y, por lo tanto, recuperan un funcionamiento y un aspecto natural, siendo comparables a aquellos bosques exentos de toda influencia humana. Presentan, por lo tanto, características funcionales (complejidad de cadenas tróficas en particular) y de diversidad biológica (especies autóctonas de gran valor biológico) parecidas a aquéllas de los estados naturales, es decir, poco perturbadas por el ser humano (SPEIGHT, 1989; BLANDIN, 1995). El grado de conservación puede ser analizado siguiendo muchos criterios, relativos esencialmente a la continuidad del estado forestal en el tiempo y del estado de madurez de las poblaciones: la antigüedad de la población forestal, su funcionalidad, la integridad de las redes tróficas, la heterogeneidad de la estructura, las poblaciones nativas (en particular la originalidad de las especies y su regeneración natural) y la biodiversidad.

Este estado próximo a la naturalidad original está caracterizado, particularmente, por el retorno de procesos silvigenéticos originales, raros en los bosques explotados, con la aparición en la cubierta forestal de fases de degradación (o colapso) y de fases de innovación, que se encuentran en el origen de un mosaico inestable y complejo de estadíos forestales variados (SCHNITZLER-LENOBLE, 2002; ANGERS et al., 2005) que permiten asegurar la existencia de muchas especies animales y vegetales, independientemente de sus exigencias ecológicas (SCHNITZLER-LENOBLE, 2002). Entre los grupos taxonómicos más favorecidos, se pueden citar, en particular, las aves (PICHARD, 1998; BLONDEL, 2005), los insectos, particularmente los coleópteros saproxílicos (BOUGET \& GOSSELIN, 2005; BRUSTEL \& DODELIN, 2005), los hongos (DAILLANT, 1996; RICHARD et al., 2005), los quirópteros y los micromamíferos (TILLION, 2005). Muchos autores (FALINSKI, 1986; KOOP, 1989; CARBIENER, 1995; PETERKEN, 1996; KEMPF, 1997) recuerdan que son estos bosques viejos los que representan, entre todos los medios naturales, la diversidad biológica más importante, lo que permite considerarlos como ecosistemas excepcionales (en el sentido de BERGERON, BOUCHARD \& VILLENEUVE, 1997).

Para explicar estos datos, hay que destacar algunas características fundamentales de estos bosques maduros:

- edad de los árboles más viejos (que da una idea de la edad mínima de la cobertura forestal) cerca de la longevidad de la especie en condiciones bioclimáticas y estacionales similares

- estructura caracterizada por una estratificación importante de la cubierta (FALINSKI, 1986; OLDEMAN, 1990)

- diversidad de especies leñosas mayor que en un bosque explotado, integrando especies "no comerciales" y especies restringidas a estadíos silvigenéticos específicos de los bosques viejos (FALINSKI, 1986; CARBIENER \& SCHNITZLER, 1990; ONF, 1998; LOMBARDINI, 2004)

- aumento importante de volumen, de 600 a 1000 $\mathrm{m}^{3} /$ ha en estación favorable (UUTTERA et al., 1997 ; BROWN et al., 1997 ; LEE et al., 2000), donde un bosque gestionado económicamente no comporta más que algunas centenas en las poblaciones de más edad

- proporción significativa de madera muerta en varios estados de descomposición (Figura 1), generalmente comprendida entre 10 et $200 \mathrm{~m}^{3} /$ ha (CHRISTENSEN et al. 2005 ; THEAUD, 2006), pero que puede alcanzar más de 500 $\mathrm{m}^{3} /$ ha (CHRISTENSEN et al., 2005), versus un poco más de $2 \mathrm{~m}^{3} /$ ha de media en bosques explotados (IFN, 2006) 


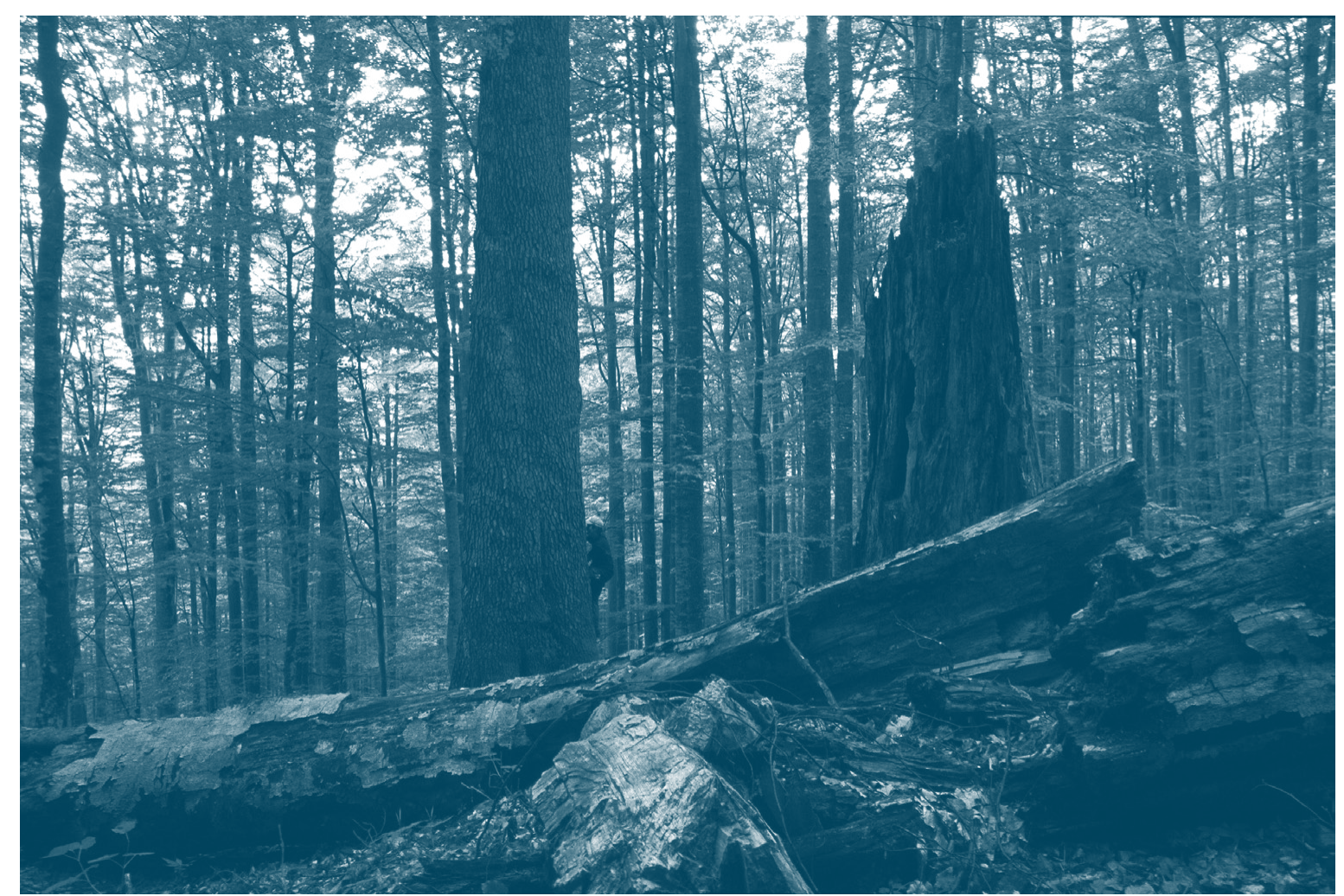

Figura 1: Madera muerta en suelo o en pie como indicador de mardurez del bosque.

- gran número de troncos muertos, particularmente de tallos de gran diámetro; la superficie basal de los árboles muertos puede representar, según las especies, del 10 al 40\% de la población (VILLENEUVE \& BRISSON, 2003)

- elevada frecuencia de defectos en los árboles: cavidades, grietas, etc. (BLONDEL, 2005) que pueden convertirse en microhábitats particulares para la fauna saproxílica.

Además, estos bosques viejos son los testigos del funcionamiento original de los bosques y, por lo tanto, representan el modelo más apropiado para mejorar el conocimiento sobre el funcionamiento de los ecosistemas forestales en general (KOOP, 1989; SCHNITZLER-LENOBLE, 2002). Los sitios de elevada naturalidad representan patrimonios raros y útiles para la comprensión de los procesos naturales o de las especies que siguen existiendo (CARBIENER, 1996). Además, los bosques viejos constituirían reservas de diversidad genética y de adaptabilidad a las variaciones de las condiciones del medio para las especies de árboles que los forman. La diversidad genética de las poblaciones de árboles tiende a aumentar durante su envejecimiento (MOSSELER et al., 2003a, MOSSEL et al., 2003b). Esto se traduce en importantes beneficios en términos de dispersión y adaptación de las especies arbóreas de los paisajes forestales cada vez más fragmentados por las actividades humanas y sujetas a los riesgos de los cambios climáticos rápidos y a la llegada de nuevas plagas y enfermedades. La preservación de los bosques viejos, incluye por lo tanto, la problemática reciente de conservación de los recursos genéticos y la del mantenimiento de las posibilidades de captura de carbono que conciernen tanto a los naturalistas, como a los gestores forestales y a los responsables políticos. Estos bosques antiguos también deben ser una preocupación, como reservorios de biodiversidad para las políticas públicas derivadas de las leyes Grenelles: "Tramas Verde y Azul" (Programa Nacional para la conexión de áreas naturales y la circulación de especies salvajes) y "Estrategia para la Creación de Áreas Protegidas." 
DETERMINANTES DE LA PRESENCIA DE INSECTOS SAPROXÍLICOS ASOCIADOS A LOS BOSQUES VIEJOS

Los "boques viejos" ofrecen las condiciones necesarias para algunos organismos saproxílicos, coleópteros y hongos en particular y a su vez, estos organismos de exigencias y sensibilidad variadas, pueden llegar a ser indicadores útiles para la lectura de la calidad biológica de los bosques y su naturalidad (Brustel, 2004).

La población nativa de las especies de árboles y de la flora asociada a los ambientes forestales puede, determinar desde los grupos de especies particulares de fitófagos - tales como el gorgojo Rutidosoma fallax que vive sobre Oxalis acetosella (BUSE, 2011), hasta el conjunto de saproxilófagos asociados a especies típicamente forestales como el abeto o el haya. Esta particularidad vegetal es para algunos autores (por ejemplo, KIRBY 1988) el principal motivo para hablar de "naturalidad" forestal. En realidad, los bosques viejos explotados por el hombre de forma regular e intensa, pueden presentar ricas biocenosis pero, sin madera vieja y gruesa, la fauna se limita a los taxones termófilos poco exigentes respecto a la madera muerta o a los ambientes típicamente forestales.

Diversas especies poco tenidas en cuenta en explotaciones forestal como el tilo, el álamo temblón, o algunos pioneros (sauce, abedul, etc.), son muy ricas en coleópteros saproxílicos (Lampra rutilans, Saperda octopunctata, Oplosia fennica, Stenostola spp., Isorhipis marmottani, etc.). Al igual que los fitófagos primarios, estas especies de insectos asociados a especies arbóreas distribuidas irregularmente, parecen relativamente móviles y capaces de responder favorablemente a la oferta masiva de recursos de una oquedad formada tras una tormenta (BOUGET 2004), siempre que existan refugios adecuados en el paisaje de los alrededores, del orden de varios kilómetros.

La madurez de los árboles: es un punto clave para la oferta de diferentes microhábitats, tanto para los hongos como para la entomofauna saproxílica. Esta característica se aprecia de forma diferente para cada especie o población. Un bosque de ribera puede ser maduro antes de 80 años, mientras que un pino negro será naturalmente poco susceptible de acoger series saproxílicas diversificadas antes de muchos siglos. Las fases de senescencia de los árboles, que se producen en el último tercio de su ciclo silvigenético, se truncan en la mayoría de los bosques explotados, pero a veces pueden ser relativamente frecuentes en los parques artificiales o sobre los árboles y setos campestres. La entomofauna saproxílica responde muy favorablemente a la madurez de los árboles pero de acuerdo con el gremio, la madurez no es suficiente y son necesarios "áreas refugio" en el paisaje de los alrededores, es decir, en algunos cientos de metros. En el caso de los hábitats transitorios en el espacio y en el tiempo, son recursos favorables para los hongos Pleurotus y Agrocybe que crecen en la madera muerta y algunas especies (Triplax ssp., Mycetophagus spp) las detectan de lejos y las colonizan rápida y masivamente. Por el contrario, los hábitats duraderos y relativamente estables, como las cavidades, están relacionados con especies menos móviles y menos prolíficas (por ejemplo, Osmoderma eremita).

La antigüedad y la continuidad de la cobertura forestal: todas las especies no presentan la misma capacidad de dispersión y algunas no soportan dejar un ambiente forestal fresco y sombrío para colonizar una nueva entidad boscosa distante y aislada por ambientes hostiles (cultivos, zonas urbanas e incluso bosques artificiales de especies exóticas en algunos casos). Los coleópteros saproxílicos ápteros son raros y comprenden algunas especies muy rústicas, longevas, poco especializadas, etc. tales como el longicornio Morimus asper que requiere grandes troncos de madera muerta de cualquier especie arbórea. Ciertos gorgojos poco móviles de los géneros Acalles spp. y los cercanos a éste, están asociados a las ramas muertas y pueden sobrevivir en las ramas de los brezales en los páramos secundarios que han reemplazado a sus bosques originales. Otras especies como (Acalles camelus, A. echinatus o A. fallax; Ruteria hypocrita) son vistos como "fantasmas del pasado" (BUSE, 2011) en las antiguas zonas donde estaban 
presentes. Finalmente, cuando estos coleópteros son a la vez exigentes en recursos y poco móviles, se trata de poblaciones en riesgo, como algunos géneros saproxílicos ricos en especies endémicas tales como especies de Colydiidae del género Tarphius spp. del sur-oeste de la cuenca mediterránea que particularmente están diversificadas en las islas del Atlántico en los bosques de laurisilva, formación forestal heredada de la era terciaria.

\section{ALGUNOS EJEMPLOS DE COLEÓPTEROS SAPROXÍLICOS EMBLEMÁTICOS Y SU RELACIÓN CON LOS BOSQUES VIEJOS}

En Francia existen alrededor de 2500 especies de coleópteros saproxílicos y sólo 10 de ellas se benefician de un estatus legal a nivel nacional (protección, en el anexo de la directiva hábitat), por lo que representan herramientas de conservación, junto con sus recursos vitales, siempre relacionados con la madera. Sin embargo, estas 10 especies no siempre están en los bosques antiguos y algunos de éstos no cuentan con especies características de estatus que permitiría ayudar a conservarlos. De estas 10 especies, la mitad se consideran no útiles para la conservación: tres de ellas están muy localizadas y son desconocidas (Stephanopachys linearis, Stephanopachys substriatus y Rhysodes sulcatus) y la presencia actual de Phryganophilus ruficollis y Cucujus cinnaberinus en Francia está en duda.

La Rosalía, Rosalia alpina, es un coleóptero especialmente dependiente de los pies de haya muertos, pero también puede desarrollarse en los alisos o fresnos muertos, en el bosque de ribera). En la actualidad sus poblaciones están divididas en áreas discontinuas y dispersas en las montañas, llanuras y colinas (Pirineos, Alpes, Provenza, el Macizo Central, el Valle del Loira y la fachada atlántica). Su rápida respuesta a la oferta de hábitat hace que sea un buen indicador de la madurez y de la restauración de los medios boscosos en los que está presente. Su pobre representación nacional y la ausencia de otras especies asociadas a los bosques viejos mixtos y resinosos de montańa, hacen que la lista de especies cuyo hábitat debe ser protegido en Francia, sea muy limitada.

El Ciervo volante (Lucanus cervus), el Gran capricornio (Cerambyx cerdo), el Escarabajo resorte (Limoniscus violaceus) y el Escarabajo eremita (Osmoderma eremita) son buenas especies indicadoras de la calidad de la red boscosa de llanura (continuo de árboles entre los cuales hay árboles maduros con cavidades altas o bajas). Osmoderma eremita vive en grandes cavidades de árboles caducifolios maduros diversos (castaños de huertos antiguos; sauces desmochados, poda de robledales, dehesas, hayas o robles forestales muy viejos, etc.). Cerambyx cerdo coloniza las partes aéreas bien expuestas de los robles antiguos moribundos. Limoniscus violaceus lleva una vida críptica en cavidades bajas huecas y maduras de especies caducifolias o de paisajes silvipastorales heredados de larga tradición rural. Lucanus cervus se desarrolla en las partes voluminosas y muertas situadas bajo tierra de todos los caducifolios (tocones, raíces, partes de árboles vivos o restos de árboles muertos). Las investigaciones recientes han mostrado capacidads de dispersión de estas especies superiores a las que fueron medidas o se suponían, del orden de $1 \mathrm{~km}$ para Osmoderma en un solo vuelo (DUBOIS et al., 2010), y por lo menos de $2 \mathrm{~km}$ para el Ciervo volante (SINSCH \& RINK, 2007). Los trabajos recientes de Jörn Buse (BUSE et al., 2007; BUSE et al., 2008) en Alemania demuestran el carácter de "especie ingeniera de los ecosistemas de bosque" del Gran capricornio, cuyas profundas y grandes perforaciones en la madera dura de robles muertos o moribundos dan lugar a numerosos microhábitats con diferente madurez por la acción y la colonización de otros organismos (hongos, insectos, murciélagos, etc. ).

Estos elementos a los que se les añade la relativa facilidad con la que se detactan directa o indirectamente (agujeros de Capricornio, excrementos de Osmoderma), permiten el uso de estas especies para evaluar la calidad de la matriz forestal de tierras bajas o sus necesidades de restauración. Por el contrario, la protección estricta y generalizada del Gran capricornio es inadecuada, ya que está muy extendido y es localmente abundante en algunas zonas del sur de Francia. 


\section{ESTADO ACTUAL DE LOS BOSQUES MADUROS, LOS REFUGIOS DE ESPECIES EN FRANCIA}

En Francia, las masas forestales como los de Fontainebleau (Seine-et-Marne), Compiègne (Oise), Grésigne (Tarn), Sare (Pirineos Atlánticos), Sainte Baume (Bouches du Rhône), Massane (PyrénéesOrientales), representan los lugares más reconocidos de biodiversidad entomológica (BRUSTEL, 2004). Pero más debido a sus viejas particularidades de gestión y a su antigüedad, que a su estado natural. Estas zonas han sido objeto hasta el presente de un intenso uso por parte del hombre, principalmente por la tala de madera, explotación hullera y pastoreo. Estos bosques constituyen una categoría particular entre los bosques maduros: son los bosques refugio de especies en situación amenazadas (BERGERON, BOUCHARD \& VILLENEUVE, 1997). Estos bosques han sido reconocidos en muchas ocasiones como hábitats de especies para constituir sitios Natura 2000 (MEDD, 2007) y su conocimiento, aunque no completo, ya está avanzado, al menos para los sitios más extensos y más notables (BRUSTEL, 2004).

En la actualidad, los bosques con un alto grado de conservación se encuentran en las zonas altas, especialmente en los pisos montano y subalpino, más raramente en el piso de colina. Esto es debido al mayor impacto de la actividad humana en las zonas de menor altitud. La presencia de accidentes topográficos tales como barrancos y acantilados en el Verdon (Var) o la meseta de Sault (Aude) puede ser también garante de la conservación de fragmentos forestales que han estado en libre evolución durante mucho tiempo.

Las encuestas de los gestores de campo permiten identificar sitios que no han sido objeto de ninguna operación silvícola desde hace al menos 50 años, pero esta aproximación no tiene en cuenta la verdadera edad de los bosques en cuestión. Para aislar los bosques verdaderamente antiguos, de elevada conservación, es indispensable tener en cuenta el aumento de tierras de uso agrícola abandonadas en la primera mitad del siglo XX, así como recurrir a la información recogida en mapas antiguos y en archivos. La presencia de ciertas especies raras y exigentes en cuanto a sus recursos biológicos, desempeña un doble papel de alerta y de validación en este tipo de aproximación.

El Grupo de Estudio de los Bosques Antiguos de Pirineos (GEVFP) dirige un proyecto multidisciplinario - que incluye la Entomología - sobre los bosques antiguos de los Pirineos y ha estudiado de algunas zonas de bosques viejos poco conocidas: el macizo de Burat y el Bois- neuf (Haute-Garonne); el abetal de Barrada (Hautes-Pyrénées), etc. WWF hace lo mismo en el sureste, desde las montañas de la Aude, hasta los Hautes-Alpes y Córcega.

\section{ESTADO DE LA SITUACIÓN ACTUAL DE LOS BOSQUES VIEJOS, CASOS CON- TRASTADOS DE REFUGIOS DE ESPECIES SAPROXILICAS EN EL PALEARTICO OESTE}

Fuera de nuestra frontera, las situaciones similares a las de Francia u otras singulares, comienzan a ser documentadas con datos sobre los coleópteros saproxílicos. En los Cárpatos, los grandes bosques maduros rumanos, eslovacos, ucranianos o polacos, como por ejemplo Bialowieza, aunque hayan conocido siempre las actividades humanas poseen series saproxílicas extremadamente ricas, una dimensión única en los bosques de tierras bajas (llanura) del Paleártico oeste, la serie más rica de la fauna de grandes vertebrados en los refugios silvestres (linces, osos, alces, búfalos, etc.).

En los países escandinavos, y Finlandia en particular, la continuidad espacio-temporal de la cobertura forestal es la más importante de Europa, sin embargo, la fuerte presión de la silvicultura ha rejuvenecido considerablemente las poblaciones de árboles que ofrecen los nichos ecológicos para los coleópteros saproxílicos más exigentes de la madera vieja. Los coleópteros responden muy bien a las recientes operaciones de restauración (quema controlada de bosque, podas abandonadas, islotes no explotados, etc.) y a una red laxa pero ampliamente 
distribuida de bosques viejos en reserva. Especies raras consideradas al borde de la extinción (Cucujus cinnaberinus, Tragosoma depsarium, Stephanbopachys spp., etc.) recolonizan masivamente estos espacios.

En la zona mediterránea, debido a factores culturales como son la cultura relacionada con los toros y la producción del excelente jamón de bellota, se da una situación original de conservación de las series saproxílicas: las dehesas ibéricas. Bajo los alcornoques y encinas explotados, junto a otros robles caducifolios o perennes, a menudo talados, huecos y muy maduros, pastan en vastas áreas desprovistas de bosques viejos (sur, centro y oeste de la Península Ibérica) cerdos y ganado vacuno en libertad. Limoniscus violaceus, Eupotosia mirifica (Figura 2) y otros coleópteros exigentes encuentran en ellos refugio (MICÓ et al, 2010a y b).

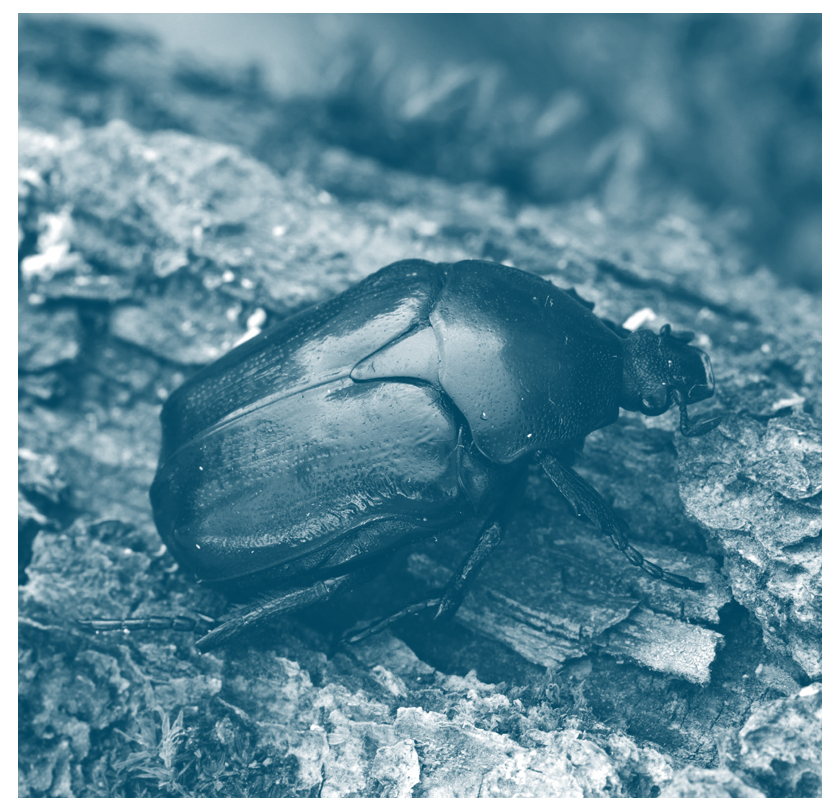

Figura 2: Eupotosia mirifica, especie vulnerable en Europa según los criterios UICN.

La Macaronesia es interesante en varios sentidos, especialmente por estas series endémicas de coleópteros saproxílicos fieles a los bosques donde han co-evolucionado durante miles de años. En las Islas Canarias, las zonas boscosas se encuentran aisladas, y ahora protegidas (es decir, son estables en superficie y están sujetas a la restauración y la maduración) y revelan fácilmente su riqueza. En Madeira, más húmedo y menos poblada antiguamente por los seres humanos, el mínimo forestal remonta probablemente a un siglo. En esta situación, la fauna es muy rica pero escasa porque los antiguos fragmentos están aislados en un inmenso bosque en recuperación. En Marruecos, por último, observamos actualmente un mínimo forestal. Las personas ya no podrán obtener beneficio del medio ambiente y sobrevivir con él. Los últimos fragmentos forestales viejos y maduros están completamente destruidos por el sobrepastoreo que ejerce una presión sobre el suelo (desnudo) y sus poblaciones arbóreas (despojadas, podadas, mutiladas). La fauna xilófaga a pesar de todo aprovecha estos escasos recursos pero la entomofauna saproxílica endémica es, por el contrario, difícil de encontrar, ¿puede en ciertos lugares haber desaparecido antes de haber sido descrita?

\section{REFERENCIAS}

ANGERS, V.A., MESSIER, C., BEAUDET, M., LEDUC, A., 2005. Comparing composition and structure in oldgrowth and harvested (selection and diameter-limit cuts) northern hardwood stand in Quebec, Forest Ecology and Management, 217, 2-3,

BARBALAT, S., 1995. Influence de l'exploitation forestière sur trois familles de coléoptères liées au bois dans les gorges de l'Areuse. Rev. Suisse Zool., 103, 2, 1-12

BARTHOD, C., 1993. La conférence des Nations Unies sur l'environnement et le développement (Rio de Janeiro, 3-14 juin 1992) et la forêt. Revue Forestière Française, $45,1,7-25$

BARTHOD, C., 1997. La protection des forêts dans la politique forestière française. Cas particulier des réserves intégrales. Communication orale, Colloque Naturalité et forêts d'Europe, Conseil de l'Europe, Strasbourg.

BARTHOD, C., TOUZET, G., 1994. De Strasbourg à Helsinki. Les deux premières conférences ministérielles pour la protection des forêts en Europe. Revue Forestière Française, 46, 4, 319-334

BERGERON, J.F., BOUCHARD, R., VILLENEUVE, N., 1997. Les écosystèmes forestiers exceptionnels du Québec, éléments-clés de la biodiversité. L’Aubelle, 117, 8-11

BLANDIN, P., 1995. Les forêts : développement ou conservation durable. Courrier de l'environnement de l'INRA, 25, 47-52 
BLONDEL, J., 2005. Bois morts et cavités : leur rôle pour l'avifaune cavicole. In Bois mort et cavités, une clé pour des forêts vivantes, Tec\&Doc

BOUGET, C., 2004. Chablis et diversité des coléoptères en forêt feuillue de plaine : impact à court terme de la trouée, de sa surface et de son contexte paysager. Thèse, Cemagref, enesad, Museum d'Histoire Naturelle (Paris) : 452 p. + annexes.

BOUGET, C., GOSSELIN, F., 2005. Distribution spatiale $\mathrm{du}$ bois mort : enjeux pour la conservation des espèces cavicoles et saproxyliques. In Bois mort et cavités, une clé pour des forêts vivantes, Tec\&Doc

BRATTON, J., ANDREWS, J., 1991. Wildlife habitat management. Invertebrate conservation. Principles and their application to broad-leaved woodland. British Wildlife, 2, 335-344

BROWN, S., SCHOEDER, P., BIRDSEY, R., 1997. Aboveground biomass distribution of US eastern hardwood forests and the use of large trees as indicator of forest developpement, Forest Ecology and Management, 96, $1-2,37-47$

BRUSTEL, H., 2004. Coléoptères saproxyliques et valeur biologique des forêts françaises. Les dossiers forestiers de l'ONF, 297 p.

BRUSTEL, H., DODELIN, B., 2005. Coléoptères saproxyliques : exigences biologiques et implications de gestion. In Bois mort et cavités, une clé pour des forêts vivantes, Tec\&Doc

BURGMAN, M.A., LINDENMAYER, D.B., 1998. Conservation biology for the Australian environnement. Surrey Beatty \& Sons, 380 p.

BÜSE, J., SCHRÖDER, B., ASSMANN, T., 2007. Modelling habitat and spatial distribution of an endangered longhorn beetle - a case study for saproxylic insect conservation. Biol Conserv 137 : 372-381

BÜSE, J., RANIUS, T., ASSMANN, T., 2008. An endangered longhorn beetle associated with old oaks and its possible role as an ecosystem engineer. Conservation Biology, 22 (2): 329-337.

BÜSE, J., 2011. "Ghosts of the past ": flightless saproxylic weevils (Coleoptera, Curculionidae) are relict species in ancient woodlands. Published online. J Insect Conserv. Original paper. P 1-5.
CARBIENER, D., 1995. Les arbres qui cachent la forêt. La gestion forestière à l'épreuve de l'écologie. Edisud, Aix-en-Provence

CARBIENER, D., 1996. Pour une gestion écologique des forêts européennes. Courrier de l'environnement de l'INRA, 29, 19-38

CARBIENER, R., SCHNITZLER, A., 1990. Evolution of major pattern models and processes of alluvial forests of the Rhine in the rift valley (France/Germany). Vegetatio, 88, 115-129

CHRISTENSEN, M., HAHN, K., MOUNTFORD, E., ODOR, P., STANDOVAR, T., ROZENBERGAR, D., DIACI, J., WIJDEVEN, S., MEYER, P., WINTER, S., VRSKA, T., 2005. Dead wood in European beech forest reserves. Forest Ecology and Management,

CINOTTI, B., 1996. Évolution des surfaces boisées en France : proposition de reconstitution depuis le début du XIXe siècle, Revue forestière française, XLVIII, 6, 547-562.

DAILLANT, O., 1996. Espèces indicatrices de forêts anciennes. Bulletin de l'Observatoire Mycologique, 9, 11-12

DUBOIS GF, LE GOUAR PJ, DELETTRE YR, BRUSTEL H \& VERNON P. 2010. Sex-biased and body condition dependent dispersal capacity in the endangered saproxylic beetle Osmoderma eremita (Coleoptera: Cetoniidae). Journal of Insect Conservation. (accepté 3/2010) published online 30/05/2010

DUPOUEY, J.L., DAMBRINE, E., LAFFITE, J.D., MOARES, C., 2006. Irreversible impact of past use on forest soils and biodiversity. Ecology, 83, 11, 2978-2984

FALINSKI, J.B., 1986. Vegetation dynamics in temperate lowland primeval forests. Dr. W. Junk Publishers, GoeBotany, 8.

IFN, 2006. Résultats des inventaires forestiers départementaux. www.ifn.fr

KEMPF, C., 1997. Bialowieza, forêt vierge d'Europe. Setec, Bialystok.

KIRBY, K.J., 1988. A woodland survey handbook. Nature conservation committee, Research and survey in nature conservation, 11, $164 \mathrm{p}$.

KOOP, H., 1989. Forest dynamics. Silvistar : a comprehensive monitoring system. Springer-Verlag, Berlin, 229 p. 
LEE, P., HANUS, S., GROVER B., 2000. Criteria for estimating old-growth in boreal mixedwood from standard timber inventory. Forest Ecology and Management, $129,1-3$,

LOMBARDINI, F., 2004. Etude préalable à la mise en place d'une réseau de forêts en vieillissement sur le territoire du parc du Lubéron. Définition des critères de sélection des peuplements ; identification des outils réglementaires et élaboration d'un protocole de suivi scientifique. Mémoire de fin d'études FIF, Nancy.

MICÓ, E., BRIONES, R., QUINTO, J., GALANTE, E. 2010. Presencia de Eupotosia mirifica (Mulsant, 1842) en la Reserva Campanarios de Azaba, Salamanca (LIFE Nature) (Coleoptera: Scarabaeoidea: Cetoniidae, Cetoniini). Boln. Asoc. esp. Ent., 34 (3-4): 437-440,

MICÓ, E., QUINTO, J., BRIONES, R., BRUSTEL, H., GALANTE, E. 2010. Nueva cita de Limoniscus violaceus (Müller, 1821) (Coleoptera: Elateridae) para la región Mediterránea Ibérica. Boln. Asoc. esp. Ent., 34 (3-4): 427-429

MINISTERE DE L'ECOLOGIE, DU DEVELOPPEMENT ET DE LAMENAGEMENT DURABLES, 2007. Portail Natura 2000. http://www.natura2000.fr/

MOSSELER, A., MAJOR, J.E., RAJORA, O.P., 2003 a. Old-growth red spruce forests as reservoirs of genetic diversity and reproductive fitness. Theoret. Appl. Genet., 106, 931-937

MOSSELER, A., THOMPSON, I., PENDREL, B., 2003 b. Overview of old-growth forests in Canada from a science perspective. Environmental Review, 11, 1-7

OLDEMAN, R.A.A., 1990. Forests : elements of silvology. Springer-Verlag, Berlin. 624 p.

ONF, 1998. Arbres morts et à cavité. Guide technique. Pourquoi ? Comment?

PETERKEN, G., 1996. Natural Woodland. Ecology and conservation in northern temperate regions. Cambridge University Press.

PERERA, A., BALDWIN, D., YEMSHANOV, D., SCHNEKENBURGER, F., WEAVER, K., BOYCHUK, D., 2003. Predicting the potential for old-growth forests by spatial simulation of landscape ageing patterns. The Forestry Chronicle, 79, 3, 621-631

PICHARD, O., 1998. Elaboration d'une méthode de régénération des chênaies du plateau lorrain prenant en compte la biodiversité. Mémoire de fin d'études FIF, Nancy.
PONTHUS, C., 1996. Inventaire des forêts subnaturelles des Pyrénées françaises. Mémoire de fin d'études. ENSAT, 58 p. + ann.

RICHARD, F., CORRIOL, G., MOREAU, P.A.,. SELOSSE, M.-A, M. GARDES, M., 2005. Conservation des champignons saproxyliques en France : perspectives pour la gestion forestière. In : D. VALLAURI, J. ANDRÉ, B. DODELIN, R. EYNARD-MACHET \& D. RAMBAUD (coord.), Bois mort et à cavités. Une clé pour les forêts vivantes. Ed. Tec et Doc, p.157-165.

RINK M., SINSCH U., 2007. Radio-telemetric monitoring of dispersing stag beetles: implications for conservation. J Zool 272 : 235-243

SCHNITZLER-LENOBLE, A., 2002. Ecologie des forêts naturelles d'Europe. Biodiversité, sylvigenèse, valeur patrimoniale des forêts primaires. Tec\&Doc, $271 \mathrm{p}$.

SIPPOLA, A.L., SIITONEN, J. KALLIO, R., 1998. Amount and quality of coarse woody debris in natural and managed coniferous forests near the timberline in Finnish Lapland. Scandinavian Journal of Forest Research, 13, 204-214

SNÄLL, T., 1997. Dead wood quantity and edge effects on dead wood and fungal indicator-species, a study conducted in south-central Swedish key-habitats. Master thesis, Swedish University of Agricultural Sciences, 20 p.

SPEIGHT M.C.D., 1989. Les invertébrés saproxyliques et leur protection. Conseil de l'Europe, coll sauvegarde de la nature, $42: 1-78$.

THEAUD, R., 2006. Evaluation du protocole dendrométrique MEDD dans les réserves biologiques intégrales de Fontainebleau. Mémoire de Master, Université de Bordeaux I, $53 \mathrm{p}$.

TILLON, L., 2005. Biodiversité, dynamique et conservation des petits mammiferes cavicoles en France. In Bois mort et cavités, une clé pour des forêts vivantes, Tec\&Doc, $145-155$

UUTTERA, J., MALTAMO M., HOTANEN J.P., 1997. The structure of forest stands in virgin and managed peatlands : a comparison between Finnish and Russian Karelia. Forest Ecology and Management, 96, 1-2,

VILLENEUVE, N., BRISSON, J., 2003. Old-growth forests in the temperate deciduous zone of Quebec : identification and evaluation for conservation and research purposes. The Forestry Chronicle, 79, 3, 559-569 